\title{
Decreased hepatic phosphorylated p38 mitogen-activated protein kinase contributes to attenuation of thioacetamide- induced hepatic necrosis in diet-induced obese mice
}

\author{
Makoto Shirai, Shingo Arakawa, Munehiro Teranishi and Kiyonori Kai \\ Medicinal Safety Research Laboratories, Daiichi Sankyo Co., Ltd., \\ 1-16-13 Kitakasai, Edogawa-ku, Tokyo 134-0081, Japan
}

(Received August 27, 2015; Accepted January 5, 2016)

\begin{abstract}
We previously reported that thioacetamide (TA)-induced hepatocellular necrosis was attenuated in mice fed a high-fat diet (HFD mice) compared with mice fed a normal rodent diet (ND mice). In this study, we investigated whether p38 mitogen-activated protein kinase (p38 MAPK) was involved in this attenuation. Western blot analysis revealed that hepatic phosphorylated p38 MAPK protein decreased at 8 and 24 hours (hr) after TA dosing in the HFD mice, while it decreased only at $24 \mathrm{hr}$ in the ND mice in comparison to the time- and diet-matched, vehicle-treated mice. p38 MAPK regulates various biological functions including inflammation, therefore, hepatic metabolomics analysis focusing on pro-inflammatory lipid mediators was performed. At $24 \mathrm{hr}$ after TA dosing, only one pro-inflammatory mediator, 12-hydroxyeicosatetraenoic acid (HETE), was higher in the HFD mice. On the other hand, in addition to 12-HETE, 15-HETE and 12-hydroxyeicosapentaenoic acid (HEPE) were higher and omega-3/ omega-6 polyunsaturated fatty acids ratios were lower in the ND mice at $24 \mathrm{hr}$. These results of metabolomics indicated that less pro-inflammatory state was seen in HFD mice than in ND mice at $24 \mathrm{hr}$. Finally, to confirm whether the observed decrease in phosphorylated p38 MAPK could attenuate TA-induced hepatocellular necrosis, we showed that SB203580 hydrochloride, an inhibitor of p38 MAPK, partially attenuated TA-induced hepatic necrosis in ND mice. Collectively, these results suggest that a prompt decrease in phosphorylation of $\mathrm{p} 38$ MAPK after TA administration is one of the factors that attenuate TAinduced hepatic necrosis in HFD mice.
\end{abstract}

Key words: Mouse, p38 MAPK, High-fat diet, Thioacetamide, Necrosis, Liver

\section{INTRODUCTION}

Nonalcoholic fatty liver disease (NAFLD) is a highly prevalent disease in developed countries (Cohen et al., 2011). Histopathologically, the livers of NAFLD patients demonstrate steatosis (Cohen et al., 2011), and a similar change is noted in the livers of high fat diet-induced NAFLD model animals (Imajo et al., 2013). We previously demonstrated that thioacetamide (TA), an extensively used hepatotoxicant (Chilakapati et al., 2005), induced hepatic necrosis that was significantly attenuated in mice fed a high-fat diet (HFD mice) compared to mice fed a normal rodent diet (ND mice; Shirai et al., 2013; Shirai et al., 2015). Furthermore, we reported that suppression of TA-induced hepatic oxidative stress by activation of the synthesis of hepatic glutathione was thought to be one of the factors that attenuate TA-induced hepatic necrosis in HFD mice (Shirai et al., 2015). However, oxidative stress seemed not to be the only cause of TAinduced hepatic necrosis in mice because hepatic necrosis did not completely disappear when the mice were pretreated with antioxidant before TA dosing (Shirai et al., 2015). This means that factors other than the activated synthesis of hepatic GSH could be involved in the mechanism of the attenuated hepatic necrosis in HFD mice. In addition, compared to ND mice, HFD mice showed more severe hepatocellular swelling at $8 \mathrm{hr}$ after TA dosing and thereafter (Shirai et al., 2013, 2015). In general, swelling is histopathologically considered to represent cellular damage, and we considered that swollen hepatocytes were moderately damaged but were not damaged severely enough for necrosis to occur. Therefore, it was unclear

Correspondence: Makoto Shirai (E-mail: shirai.makoto.v7@daiichisankyo.co.jp) 
why the swelling in HFD mice was so severe at $8 \mathrm{hr}$ while hepatocellular necrosis was slight and comparable to that in the ND mice, and the necrosis in HFD mice was slighter than in ND mice at 24 and $48 \mathrm{hr}$.

p38 mitogen-activated protein kinase (MAPK) is a kinase that is activated to respond to growth factors or environmental stimuli (Kumar et al., 2003). It was previously reported that $\mathrm{p} 38$ MAPK controls cellular volume and inhibition of this enzyme results in cellular swelling (Feranchak et al., 2001; vom Dahl et al., 2001). Furthermore, p38 MAPK is also known to regulate various types of biological events such as cellular differentiation and proliferation, inflammation and apoptosis (Cuenda and Rousseau, 2007; Kumar et al., 2003; Lee et al., 2000). Although the roles of p38 MAPK in the liver have not been completely clarified, some reports indicated that p38 MAPK is involved in hepatotoxicant-induced hepatotoxicity. For example, ethanol administration elicited rapid p38 MAPK activation before hepatic necrosis occurred (Aroor et al., 2010). Likewise, activated p38 MAPK was noted after administration of lipopolysaccharide/D-galactosamine (Zhang et al., 2010) or carbon tetrachloride (Iida et al., 2009). Furthermore, inhibition of p38 MAPK activity reduced liver injury induced by ethanol plus Fas agonistic antibody (Wang et al., 2009), pyrazole plus lipopolysaccharide (Wu and Cederbaum, 2008), or hemorrhagic shock and resuscitation (Lv et al., 2012). These results suggest the possibility that the activity of p38 MAPK changes in HFD animals, and this change induces hepatocellular swelling and modifies hepatic sensitivity of the model animals against TA.

The purpose of this study was to investigate wheth- er hepatic p38 MAPK contributes to the attenuated TAinduced hepatic necrosis in HFD mice. In this study, the amounts of hepatic total p38 MAPK and phosphorylated p38 MAPK (p-p38 MAPK) protein after TA administration were compared between ND and HFD mice. Hepatic pro-inflammatory lipid mediators were also investigated to examine the influence of the changed level of p-p38 MAPK. In addition, whether a p38 MAPK inhibitor attenuated TA-induced hepatic necrosis was also evaluated in ND mice.

\section{MATERIALS AND METHODS}

\section{Chemicals}

TA and dimethyl sulfoxide were purchased from Wako Pure Chemical Industries, Ltd. (Osaka, Japan), 5-bromo-2-deoxyuridine (BrdU) was purchased from Sigma-Aldrich Co. (Tokyo, Japan), and SB203580 hydrochloride (SB) was purchased from Cayman Chemical (Ann Arbor, MI, USA).

\section{Experimental design}

In this study, two experiments were performed as described below. The timing of the treatments and the necropsies are outlined in Fig. 1.

\section{Experiment 1 (comparison of hepatic p38 MAPK expression in ND and HFD mice) \\ Liver samples}

Liver samples were collected from animals and some of the results were reported previously (Shirai et al., 2015). Briefly, three-week-old male C57BL/6J mice were

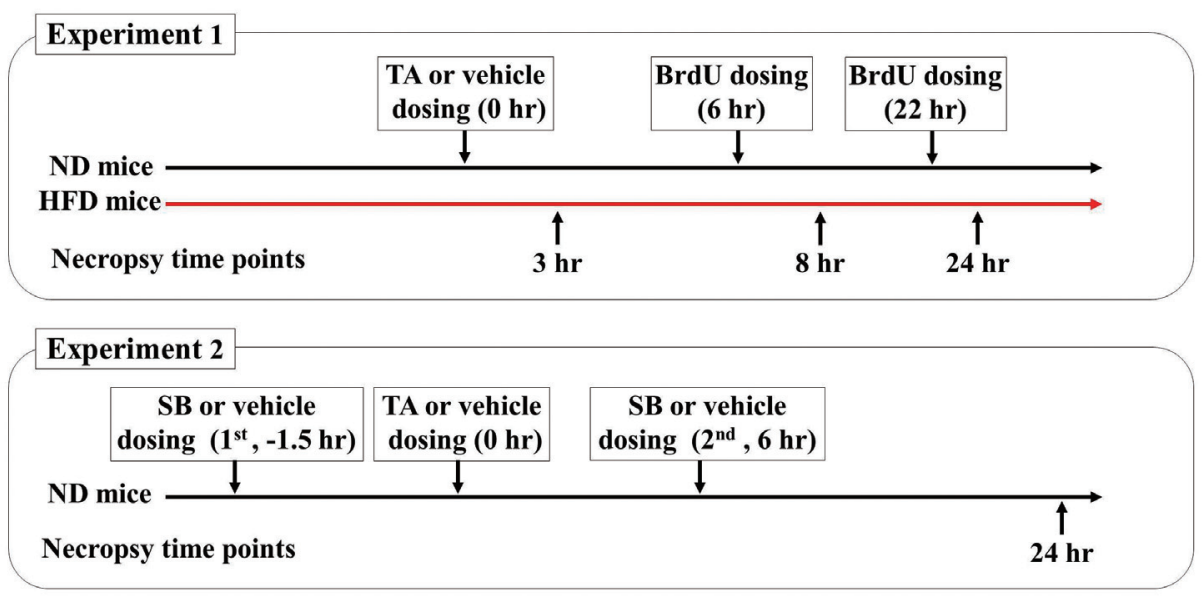

Fig. 1. Schematic diagram of experiments 1 and 2. Each diagram outlines the timing of thioacetamide (TA), 5-bromo-2-deoxyuridine (BrdU) and SB203580 hydrochloride (SB) treatments and necropsies. hr: hours. ND mice: mice fed a normal rodent diet. HFD mice: mice fed a high fat diet. 
Decreased p-p38 MAPK attenuated liver necrosis in obese mice

obtained from Charles River Japan, Inc. (Kanagawa, Japan), and were fed either a normal rodent diet (CRF-1; Oriental Yeast Co. Ltd., Tokyo, Japan) or a high-fat diet containing $60 \mathrm{kcal} \%$ fat (high fat diet 32; Clea Japan, Inc., Tokyo, Japan) and were given tap water ad libitum. After 8 weeks of feeding, all mice were given either TA (100 mg/kg body weight; dissolved in saline) or saline once intraperitoneally. The mice were necropsied at 3,8 and 24 hours (hr) after dosing. In the mice necropsied at 8 and $24 \mathrm{hr}$, BrdU (100 mg/kg body weight, dissolved with $1 \%$ dimethyl sulfoxide/saline) was given intraperitoneally $2 \mathrm{hr}$ before necropsy for additional evaluations (which will be reported separately). After euthanasia, the livers were collected, frozen in liquid nitrogen and stored at $-80^{\circ} \mathrm{C}$ in a deep freezer for the analyses described below.

\section{Western blot analysis of p38 MAPK}

For the Western blot analysis of p-p38 MAPK and total p38 MAPK (the sum of p-p38 MAPK and nonphosphorylated p38 MAPK), liver cytosol was prepared using nuclear and cytoplasmic extract reagents (NE-PER: Thermo Fisher Scientific Inc., Rockford, IL, USA). The protein concentration of the cytosol was adjusted to $10 \mathrm{mg} / \mathrm{mL}$ with phosphate-buffered saline and subsequently diluted to $5 \mathrm{mg} / \mathrm{mL}$ with Tris-SDS beta-mercaptoethanol sample loading buffer (Cosmo Bio Co., Ltd., Tokyo, Japan). Then samples were heated at $95^{\circ} \mathrm{C}$ for $5 \mathrm{~min}$, and $10 \mu \mathrm{L}(50 \mu \mathrm{g})$ of each sample was loaded onto $10 \%$ polyacrylamide gel (Funakoshi Corporation, Tokyo, Japan) and subjected to electrophoresis. The proteins were transferred from the gel to an Immobilon ${ }^{\circledR}$ polyvinylidene difluoride membrane (Millipore Corporation, Billerica, MA, USA) using a blotting apparatus (Horizeblot: Atto Corporation, Tokyo, Japan). This membrane was blocked with enhanced chemiluminescence (ECL) blocking agent (GE Healthcare, Buckinghamshire, UK) and treated successively with primary antibodies. Anti-human p-p38 MAPK (Thy 180/Tyr 182) and p38 MAPK antibodies (Cell Signaling Technology, Danvers, MA, USA) were used as primary antibodies. Then, the membrane was treated with biotin-labeled anti-rabbit IgG antibody (GE Healthcare) as the secondary antibody and finally treated with streptavidin-horseradish peroxidase conjugate (GE Healthcare). Protein-antibody complexes were detected using ECL Western blotting detection reagent (GE Healthcare) and the membrane was exposed to instant film (Fujifilm Corporation, Tokyo, Japan). The protein bands were scanned with AE-6961 (Atto Corporation, Tokyo, Japan) and the intensity was quantified with densitometry analyzer (CS
Analyzer, Atto Corporation). The results of the quantification were expressed as percentage of the value of the vehicle-treated ND mice at each time point.

\section{Metabolomics analysis of pro-inflammatory lipid mediators in the liver}

To investigate inflammatory state in the liver, a metabolomics analysis was performed at Metabolon Inc. (Durham, NC, USA). The results regarding glutathione metabolism was reported previously (Shirai et al., 2015). In brief, the samples from the frozen liver were prepared using an automated MicroLab STAR ${ }^{\circledR}$ system (Hamilton Company, Salt Lake City, UT, USA). The samples were then separated into three equal aliquots for analysis in three independent platforms: ultra-high performance liquid chromatography/tandem mass spectrometry (UHPLC/ MS/MS) optimized for basic species, UHPLC/MS/MS optimized for acidic species, and gas chromatography/ mass spectrometry (GC/MS). Metabolites were identified by automated comparison of the ion features in the experimental samples with a reference library of purified standards; these features included retention time, molecular weight $(\mathrm{m} / \mathrm{z})$, preferred adducts, and in-source fragments as well as their associated MS/MS spectra. Among the identified metabolites, metabolites relating to pro-inflammatory lipid mediators were investigated.

\section{Experiment 2 (the effect of p38 MAPK inhibitor SB on TA-induced hepatotoxicity in ND mice)} In-Life experiments and histopathological examination

Eight-week-old male C57BL/6J mice were obtained from Charles River Japan, Inc., and were fed a normal rodent diet. At 11 weeks of age, all of the mice were given either TA $(100 \mathrm{mg} / \mathrm{kg})$ or saline once intraperitoneally as described in Experiment 1. To inhibit hepatic p38 MAPK function, the mice were given SB $(75 \mathrm{mg} / \mathrm{kg}$ body weight; dissolved with saline) or saline twice intraperitoneally, once at $1.5 \mathrm{hr}$ before and again at $6 \mathrm{hr}$ after TA or saline administration in reference to the previous reports, in which in vivo p38 MAPK inhibition was performed by SB (Tibbles et al., 2002; Cao et al., 2004; Badger et al., 1996). Hence, there were 4 groups, vehicle-only treated group (vehicle/vehicle group), SB- and vehicle-treated group (SB/vehicle group), vehicle- and TA-treated group (vehicle/TA group) and SB- and TA-treated group (SB/ TA group). All of the mice were necropsied at $24 \mathrm{hr}$ after administration, and the left lateral lobe and right and left medial lobes of the liver were trimmed, fixed in $10 \%$ neutral buffered formalin and embedded in paraffin for histopathology. 


\section{Histopathological examination}

Histopathological specimens were prepared and stained with hematoxylin and eosin (HE). As reported previously (Shirai et al., 2013, 2015), TA-induced centrilobular necrosis of hepatocytes and centrilobular swelling of hepatocytes were graded as described below.

Centrilobular necrosis of hepatocytes

Grade 1: Centrilobular necrosis that reached zone 2 was observed in less than 5 lobules in the 3 lobes (the left lateral, right medial and left medial lobes).

Grade 2: Centrilobular necrosis that reached zone 2 was observed in 5 or more lobules, or centrilobular necrosis that reached zone 1 was observed in less than 5 lobules in the 3 lobes.

Grade 3: Centrilobular necrosis that reached zone 1 was observed in 5 or more lobules in the 3 lobes, and the necrotic area was less than $33 \%$ of 1 or more of the 3 lobes.

Grade 4: The necrotic area was $33 \%$ to $66 \%$ of 1 or more the 3 lobes.

Grade 5: The necrotic area was more than $66 \%$ of 1 or more of the 3 lobes.

Centrilobular swelling of hepatocytes

Grade 1: Centrilobular swelling was observed, but it was limited to the centrilobular area in the hepatic lobe (zone 3 ).

Grade 2: Centrilobular swelling that reached zone 2 was observed in less than $50 \%$ of lobules.

Grade 3: Centrilobular swelling that reached zone 2 was observed in $50 \%$ or more of lobules.

\section{Ethics}

All of the studies were approved by the Ethics Review Committee for Animal Experimentation of Daiichi Sankyo Co., Ltd., and were conducted in compliance with the "Law Concerning the Protection and Control of Animals" (Japanese Law 105, October 1, 1973; revised on June 22, 2005).

\section{Statistical analysis}

The results are expressed as the mean \pm S.D. In analysis of the Western blot data, the F-t test was performed. The homogeneity of variance was evaluated by the $\mathrm{F}$ test, and the Student's t test (for homogeneous data) or the Aspin-Welch t test (for heterogeneous data) was performed using Microsoft Office Excel 2003 (Microsoft, Redmond, WA, USA). In analysis of histopathological data, the Mann-Whitney U test was performed using the common gateway interface program (http://aoki2. si.gunma-u.ac.jp/exact/utest/getpar.html). In analysis of metabolomics data, a log transformation was applied to the observed relative concentrations for each metabolite because, in general, the variance increases as a function of the average response of the metabolite. After log transformation, a three-way ANOVA was performed using Array Studio (Omicsoft Corp., Cary, NC, USA) and/or "R" (http://cran.r-project.org/), and multiple comparisons were performed with the false discovery rate (FDR) method. Each FDR was estimated using the q-values. Statistical analysis was not performed on the ratios of arachidonate/eicosapentaenoate and linoleate/eicosapentaenoate. We used the conservative criteria of $\mathrm{p}<0.05$ and $\mathrm{q}<0.05$ to judge statistical significance in all statistical analyses.

\section{RESULTS}

\section{P-p38 MAPK and total p38 MAPK protein expression levels in HFD and ND mice (Experiment 1)}

We previously reported that, severe hepatocellular necrosis was noted in the ND mice at 24 and $48 \mathrm{hr}$, but hepatocellular necrosis was significantly attenuated in the HFD mice (Shirai et al., 2015). To examine whether p38 MAPK was involved in this attenuation in the HFD mice, the amounts of total p38 MAPK and p-p38 MAPK protein were measured by Western blot analysis. In the TAdosed HFD mice, p-p38 MAPK almost disappeared at $8 \mathrm{hr}$ after dosing (Fig. 2). At 24 hr, p-p38 MAPK apparently diminished in both the TA-dosed ND mice and HFD mice. Densitometry analysis revealed that the amount of p-p38 MAPK in the TA-administered HFD mice was significantly lower than that of the vehicle-treated HFD mice or that of the TA-treated ND mice at $8 \mathrm{hr}$ (Fig. 3A). There

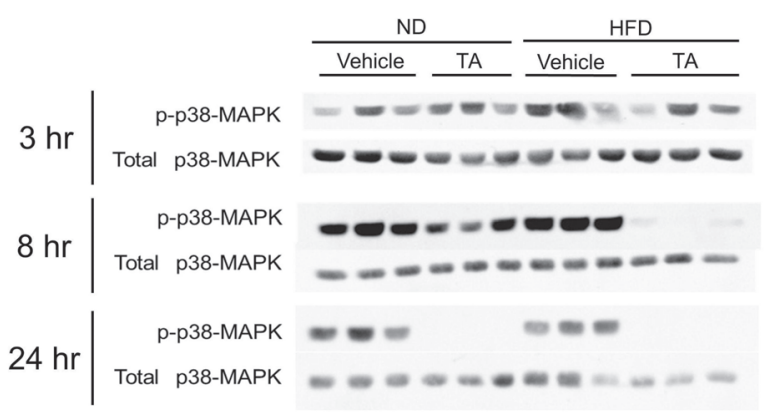

Fig. 2. Western blots of hepatic total p38 MAPK and phospho-p38 MAPK in the livers of the vehicle- and TAtreated mice. The number of mice fed the high-fat diet (HFD) or normal rodent diet (ND) was 3 at each observation time. hr: hours. p-p38 MAPK: phospho-p38 MAPK. 
A

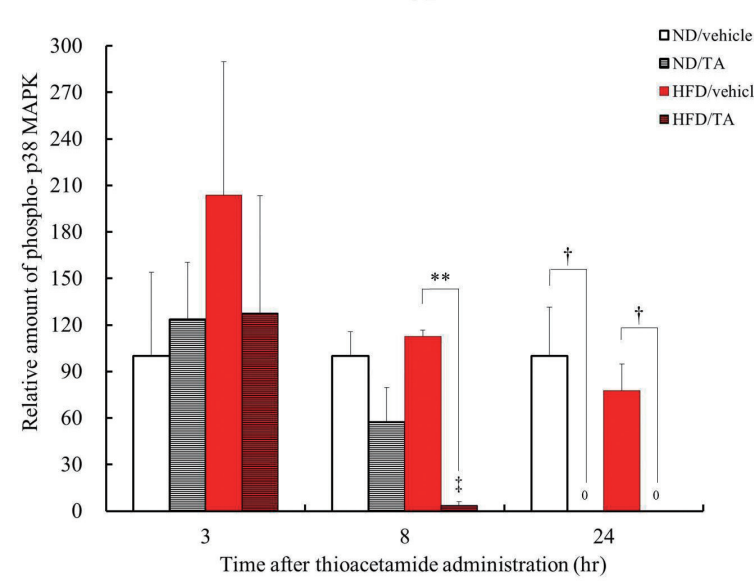

B

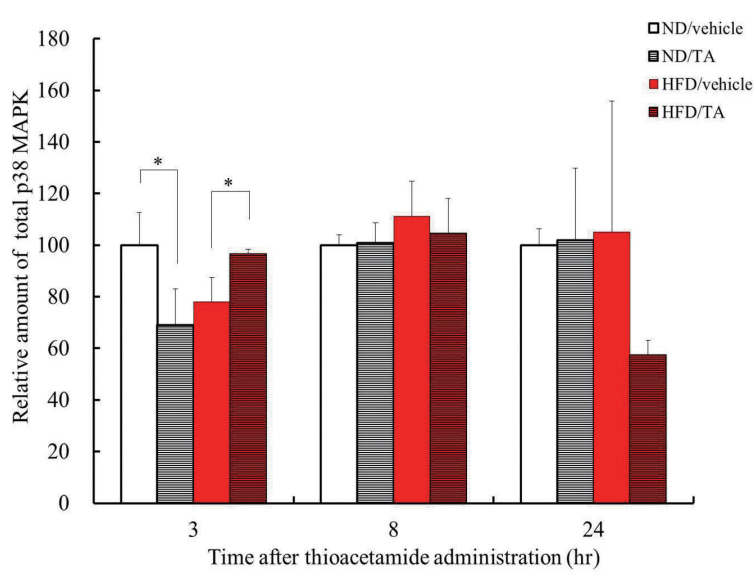

Fig. 3. The relative amounts of phospho p38 MAPK (A) and total p38 MAPK 8 (B) are shown in Fig. 2. The amounts of these proteins were calculated by densitometry analysis and the amounts were expressed as percentages of the value of the vehicle-treated ND mice at each time point. The number of mice fed the high-fat diet (HFD) or normal rodent diet (ND) was 3 at each observation time. White columns: vehicle-treated ND mice. White columns with black shading: TA-dosed ND mice. Red columns: vehicle-treated HFD mice. Red columns with black shading: TA-dosed HFD mice. $*, * *: p<0.05$ and $\mathrm{p}<0.01$ for comparison between the vehicle-treated ND mice and the TA-dosed ND mice, or between the vehicle-treated HFD mice and the TA-dosed HFD mice (student's t-test). $\uparrow: p<0.05$ for comparison between the vehicle-treated ND mice and the TA-dosed ND mice, or between the vehicle-treated HFD mice and the TA-dosed HFD mice (Aspin-Welch t-test). $\$: p<0.05$ for comparison between the TA-dosed ND mice and the TA-dosed HFD mice (Aspin-Welch t-test).

was no significant difference between TA-dosed ND mice and vehicle-treated ND mice at this time point. The p-p38 MAPK blots of the TA-dosed ND and HFD mice were not detected at $24 \mathrm{hr}$ (Fig. 3A). As for total p38 MAPK, at $3 \mathrm{hr}$, significantly lower expression in the TA-dosed ND mice and significantly increased expression in the TA-dosed HFD mice were observed compared with the vehicle-treated ND mice and vehicle-treated HFD mice, respectively (Fig. 3B). At each time point, there were no statistical differences between the vehicle-treated ND and HFD mice for the amounts of either p-p38 MAPK or total p38 MAPK (Fig. 3A and 3B).

\section{Metabolomics analysis of pro-inflammatory lipid mediators in the liver (Experiment 1)}

Table 1 shows the results of the metabolomics analysis on unsaturated fatty acids and their metabolites, which have been reported to relate to pro-inflammation state (Tam, 2013; Cunningham et al., 1985; Calder, 2009; Pilkington et al., 2014; Strassburg et al., 2012; Elabdeen et al., 2013; Kalogeropoulos et al., 2010). These mediators were measured at 8 and $24 \mathrm{hr}$ after TA administration.

At $8 \mathrm{hr}$ after TA dosing, 12-HETE was significantly higher in the HFD mice compared to the vehicle-treated
HFD mice. At $24 \mathrm{hr}$, 12-HETE was significantly higher in both the HFD and ND mice compared with the vehicletreated ND mice and vehicle-treated HFD mice, respectively. In addition, 15-HETE and 12-HEPE were higher and eicosapentaenoate, linoleate and arachidonate were significantly lower in the ND mice. Furthermore, the ratios of eicosapentaenoate/linoleate and eicosapentaenoate/arachidonate were apparently lower only in the ND mice, while they were higher or the same in the HFD mice at $24 \mathrm{hr}$.

\section{The effect of treatment with p38 MAPK inhibitor SB on TA-induced hepatic necrosis in ND mice (Experiment 2)}

To investigate whether $\mathrm{p} 38$ MAPK attenuates TA-induced hepatic necrosis in mice, a selective p38 MAPK inhibitor, SB, was given to the ND mice either by itself or together with TA. With SB treatment, none of the ND mice died by $24 \mathrm{hr}$ after TA dosing, while 3 out of 9 ND mice given TA without SB died (Table 2). Moreover, although TA induced centrilobular hepatocellular necrosis with or without SB treatment, SB significantly attenuated the severity of TA-induced hepatic necrosis at $24 \mathrm{hr}$ (Figs. $4 \mathrm{~A}$ and $4 \mathrm{~B}$, Table 2). 
Table 1. Hepatic pro-inflammatory lipid mediators.

\begin{tabular}{|c|c|c|c|c|c|c|c|c|c|c|}
\hline \multirow[b]{3}{*}{ Biochemical Name } & \multirow[b]{3}{*}{ KEGG } & \multirow[b]{3}{*}{ HMDB } & \multicolumn{4}{|c|}{$8 \mathrm{hr}$} & \multicolumn{4}{|c|}{$24 \mathrm{hr}$} \\
\hline & & & \multicolumn{2}{|c|}{ ND } & \multicolumn{2}{|c|}{ HFD } & \multicolumn{2}{|c|}{ ND } & \multicolumn{2}{|c|}{ HFD } \\
\hline & & & vehicle & TA & vehicle & TA & vehicle & TA & vehicle & TA \\
\hline Eicosapentaenoate $(20: 5 \mathrm{n} 3)$ & & & 4.4733 & 4.479 & 0.5043 & 0.3287 & 7.1776 & $1.1081^{*}$ & 0.5694 & 0.6777 \\
\hline Linoleate (18:2n6) & & & 0.8808 & 1.0202 & 0.9648 & 0.8172 & 1.2018 & $0.6707^{* *}$ & 1.0004 & 1.197 \\
\hline Arachidonate $(20: 4 \mathrm{n} 6)$ & & & 0.8244 & 0.9639 & 2.0286 & 1.5333 & 0.7994 & $0.4497^{* *}$ & 1.9545 & 1.7596 \\
\hline 5-HETE & & & 1.3093 & 1.0107 & 0.5728 & 0.5633 & 1.5248 & 2.7212 & 0.8697 & 0.9554 \\
\hline 12-HETE & & HMDB06111 & 0.9185 & 1.3386 & 0.4028 & $0.9371^{* *}$ & 0.8294 & $6.7603^{* *}$ & 0.5735 & $3.8228^{* *}$ \\
\hline 15-HETE & $\mathrm{C} 04742$ & HMDB02110 & 0.9736 & 0.9003 & 0.7659 & 0.6818 & 1.1193 & $2.7601^{*}$ & 0.8332 & 1.2954 \\
\hline 12-HEPE & & HMDB10202 & 1.3675 & 1.5867 & 0.6441 & 0.7395 & 1.6396 & $5.2847^{* *}$ & 0.6714 & 0.8476 \\
\hline Eicosapentaenoate/linoleate ratio & & & 5.08 & 4.39 & 0.52 & 0.4 & 5.97 & 1.65 & 0.57 & 0.57 \\
\hline Eicosapentaenoate/arachidonate ratio & & & 5.43 & 4.65 & 0.25 & 0.21 & 8.98 & 2.46 & 0.29 & 0.39 \\
\hline
\end{tabular}

$8 \mathrm{hr}$ and $24 \mathrm{hr}$ : Animals necropsied at 8 or $24 \mathrm{hr}$ after vehicle or thioacetamide (TA) administration, respectively $(\mathrm{n}=5)$. ND: mice fed a normal rodent diet, HFD: mice fed a high fat diet. KEGG: Kyoto Encyclopedia of Genes and Genomes, HMDB: Human Metabolome Database. Each value was re-scaled to have median equal to 1. Statistical analysis was not performed on eicosapentaenoate/linoleate and eicosapentaenoate/arachidonate ratios. ${ }^{*}{ }^{* *}: \mathrm{p}<0.05$ or $\mathrm{p}<0.01$ in the value of each metabolite versus time-matched vehicle-treated group (three-way ANOVA).

Table 2. The effect of SB203580 hydrochloride administration on thioacetamide-induced hepatic lesions at $24 \mathrm{hr}$ after dosing in mice fed a normal rodent diet.

\begin{tabular}{|c|c|c|c|c|}
\hline Hours after TA administration & vehicle/vehicle & SB/vehicle & vehicle/TA & SB/TA \\
\hline \multicolumn{5}{|l|}{ Mice Fed a Normal Rodent Diet } \\
\hline Number of mice a & $6(0)$ & $6(0)$ & $9(3)$ & $9(0)$ \\
\hline Incidence of necrosis ${ }^{b}$ & $6,0,0,0,0,0$ & $6,0,0,0,0,0$ & $0,0,0,0,6,3$ & $0,0,2,5,1,1$ \\
\hline Mean score & $\mathbf{0 . 0} \pm \mathbf{0 . 0 0}$ & $\mathbf{0 . 0} \pm \mathbf{0 . 0 0}$ & $4.3 \pm 0.50$ & $3.1 \pm 0.93^{* *}$ \\
\hline Incidence of swelling ${ }^{b}$ & $6,0,0,0$ & $6,0,0,0$ & $9,0,0,0$ & $9,0,0,0$ \\
\hline Mean score & $\mathbf{0 . 0} \pm \mathbf{0 . 0 0}$ & $0.0 \pm 0.00$ & $0.0 \pm \mathbf{0 . 0 0}$ & $\mathbf{0 . 0} \pm \mathbf{0 . 0 0}$ \\
\hline
\end{tabular}

vehicle/vehicle group: group treated only with vehicle, SB/vehicle group: group treated with SB203580 hydrochloride (SB) and vehicle, vehicle/TA group: group treated with vehicle and thioacetamide (TA), SB/TA group: group treated with SB and TA. Mean score is the mean of grades for individual mice \pm S.D.. a: The values in parentheses show the number of dead mice. b: The numbers show the incidence of each grade ( 0 to 5 for hepatocellular necrosis and 0 to 3 for hepatocellular swelling), with the leftmost number representing grade $0 .{ }^{* *}: \mathrm{p}<0.01$ for comparison versus vehicle/TA group (Mann-Whitney U test).
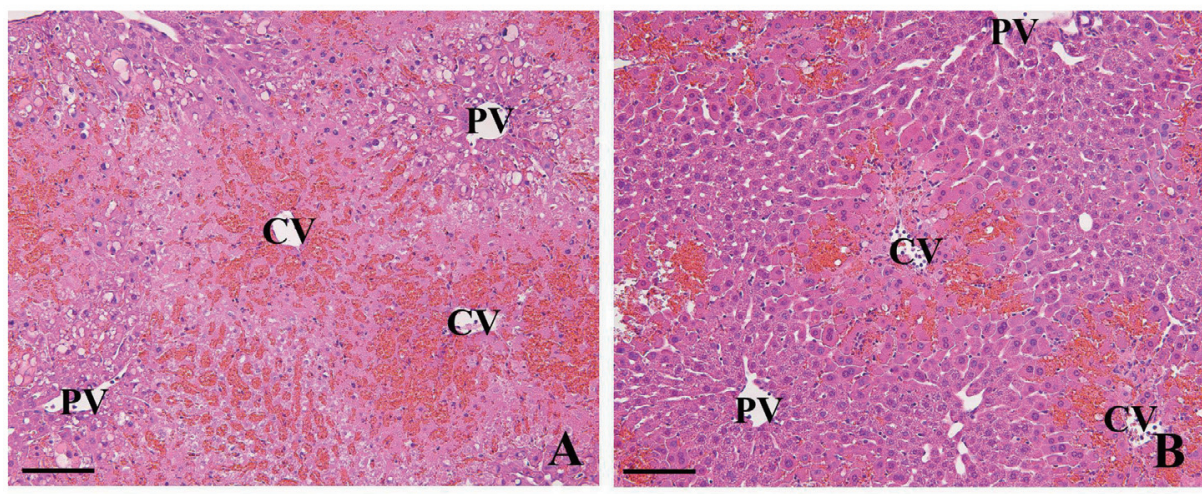

Fig. 4. Histopathological appearance of typical hepatic lesions at 24 hours after thioacetamide (TA) administration in a mouse fed a normal rodent diet. A: The liver of a mouse given TA and vehicle. B: The liver of a mouse given TA and SB203580 hydrochloride. CV: central vein, PV: periportal vein; HE stain; Bar = 100 micrometer. 
Decreased p-p38 MAPK attenuated liver necrosis in obese mice

\section{DISCUSSION}

We previously reported that severe hepatocellular necrosis was noted in ND mice at 24 and $48 \mathrm{hr}$, but hepatocellular necrosis was significantly attenuated in HFD mice (Shirai et al., 2013, 2015). On the other hand, hepatocellular swelling was noted both in ND and HFD mice, but the severity of the swelling was significantly higher in HFD mice at 8 to $48 \mathrm{hr}$. In this study, we demonstrated that p-p38 MAPK, which is the phosphorylated active form of p38 MAPK (Kumar et al., 2003), was significantly lower in the livers of the HFD mice at 8 and $24 \mathrm{hr}$ after TA administration in comparison to the vehicle-treated HFD mice at the same time point. In the ND mice, such decrease was observed only at $24 \mathrm{hr}$. p38 MAPK is a multifunctional protein kinase and its biological role is both beneficial and harmful (Cuenda and Rousseau, 2007). The function of p38 MAPK in the liver has not been fully unveiled, but it was reported that p38 MAPK seemed to be involved in hepatotoxicity induced by some hepatotoxicants (Aroor et al., 2010; Zhang et al., 2010; Iida et al., 2009). In addition, inhibition of p38 MAPK is known to attenuate hepatic injury (Lv et al., 2012; Wang et al., 2009; Wu and Cederbaum, 2008). These results suggested the possibility that decreased p38 MAPK activity promptly after TA administration could contribute to the attenuation of TA-induced hepatic necrosis in HFD mice.

p38 MAPK plays an important role in inflammation, and treatment with a 38 MAPK inhibitor decreases the inflammatory response (Kumar et al., 2003; Lee et al., 2000). To investigate whether inflammatory activity was reduced together with decreased phosphorylation of p38 MAPK protein in HFD mice after TA administration, lipid mediators related to pro-inflammation were investigated by metabolomics analysis. Compared to the vehicletreated HFD mice, at $24 \mathrm{hr}$ after TA administration, only one pro-inflammatory mediator, 12-HETE (Cunningham et al., 1985), was higher in the HFD mice. On the other hand, in addition to 12-HETE, 2 pro-inflammation-related metabolites, 15-HETE (Calder, 2009) and 12-HEPE (Pilkington et al., 2014; Strassburg et al., 2012), were higher in the ND mice in comparison to the vehicle-treated ND mice. Moreover, the omega-3 polyunsaturated fatty acid eicosapentaenoate and the omega- 6 polyunsaturated fatty acids, linoleate and arachidonate (Chen et al., 1996), were significantly lower and the ratio of omega-3/ omega- 6 polyunsaturated fatty acids was apparently lower only in the ND mice at $24 \mathrm{hr}$. It was previously reported that a decrease in the ratio of omega-3/omega- 6 polyunsaturated fatty acids may be a marker of pro-inflammation because omega- 3 and omega- 6 polyunsaturated fat- ty acids induce and reduce inflammation, respectively (Elabdeen et al., 2013; Kalogeropoulos et al., 2010). Collectively, our results demonstrated that livers of HFD mice exhibited less pro-inflammatory state than those of ND mice at $24 \mathrm{hr}$ after TA administration, and this result was consistent with the decrease in phosphorylated p38 MAPK observed only in the TA-dosed HFD mice at $8 \mathrm{hr}$.

Although our results indicated that a decrease in p38 MAPK activity attenuated TA-induced hepatic necrosis in HFD mice, there have been no investigations of the contribution of p38 MAPK activity to TA-induced hepatic necrosis in the mouse. Hence, to investigate the contribution of p38 MAPK to TA-induced hepatic necrosis in the mouse, the selective p38 MAPK inhibitor SB was administered to ND mice before TA dosing. In this study, SB treatment reduced mortality, and attenuated TA-induced hepatic necrosis in the ND mice at $24 \mathrm{hr}$. Our results showed that hepatic p38 MAPK activity was one of the causes of TA-induced hepatic necrosis in mice, and therefore, promptly decreased phosphorylation of p38 MAPK protein after TA administration was thought to contribute to the attenuation of TA-induced hepatic necrosis in HFD mice.

In the previous study, we demonstrated that the HFD mice exhibited more severe hepatocellular swelling after TA administration at $8 \mathrm{hr}$ (Shirai et al., 2013, 2015). Swelling is induced by osmotic imbalance and is histopathologically considered to be a toxic change. However, our previous study demonstrated that, in comparison to the ND mice, hepatocellular necrosis was slighter in the HFD mice at $24 \mathrm{hr}$ or thereafter (Shirai et al., 2015). In addition, one kind of biosyntheses, glutathione synthesis, was more active in the livers of the HFD mice at $8 \mathrm{hr}$. These results indicated that this severe swelling observed in the HFD mice at $8 \mathrm{hr}$ was not a simple and temporal severe toxic change, but was a change occurring by not only TA toxicity but also some additional biological reactions. It was reported that p38 MAPK inhibition delayed and diminished volume regulatory $\mathrm{K}(+)$ efflux and induced swelling of hepatocyte under hypoosmotic exposure (vom Dahl et al., 2001). Furthermore, p38 MAPK inhibition increased $\mathrm{Na}^{+}$currents through cell membranes and enlarged cell volume in HTC rat hepatoma cell line (Feranchak et al., 2001). p38 MAPK inhibition resulted in cellular swelling, therefore, there was a possibility that TA-induced hepatocellular swelling in HFD mice at $8 \mathrm{hr}$ was caused, at least in part, by inhibition of p38 MAPK activity. Severe hepatocellular swelling was also noted in the HFD mice at $24 \mathrm{hr}$ and the hepatic p38 MAPK was not phosphorylated at this time point. However, the involvement of p38 MAPK at this time point seemed to 
be limited because hepatic p-p38 MAPK disappeared but hepatocellular swelling was slight in the ND mice.

As for the other changes, at $3 \mathrm{hr}$ after TA dosing, total p38 MAPK significantly decreased in the ND mice and significantly increased in the HFD mice, compared with the vehicle-treated ND and HFD mice, respectively. The cause of this increase or decrease is unknown, but these changes seemed to have little biological meaning because the active form of p38 MAPK, p-p38 MAPK, did not change either in the ND or HFD mice at this time point. In metabolomics analysis, the pro-inflammatory mediator 12-HETE significantly increased at $8 \mathrm{hr}$ only in the HFD mice. However, this increase was much slighter than the increase of 12-HETE in the ND and HFD mice at $24 \mathrm{hr}$. Furthermore, the other pro-inflammatory lipid mediators or the ratio of omega-3/omega- 6 polyunsaturated fatty acids did not change in the HFD mice at $8 \mathrm{hr}$. Therefore, the increase of 12-HETE noted in the TA-dosed HFD mice at $8 \mathrm{hr}$ was not considered to indicate an activated pro-inflammatory state compared with the TA-dosed HFD mice at $24 \mathrm{hr}$ or the TA dosed ND mice at 8 and $24 \mathrm{hr}$.

In this study, there were no differences in the hepatic p-p38 MAPK between the vehicle-treated ND and HFD mice at 3, 8 and $24 \mathrm{hr}$. Hence, the phosphorylation of p38 MAPK seemed not to be affected in the HFD mice. In turn, the response against TA administration differed between these types of mice. Our study indicates the possibility that the susceptibility to hepatotoxicants is modified in NAFLD due to an alteration in the status of phosphorylation of p38 MAPK following hepatotoxicant exposure. This is the first study that addresses decreased p38 MAPK activity in NAFLD animals after TA dosing.

In conclusion, it is suggested that a prompt decrease in phosphorylated p38 MAPK after TA administration is one of the factors that attenuate TA-induced hepatocellular necrosis in HFD mice.

\section{ACKNOWLEDGMENTS}

The authors would like to thank Mr. Takayuki Sato for his assistance for care of the mice, Ms Masae Yagi for her assistance for western blot analysis, and Ms. Kimiko Hara, Shinobu Hakamata and Yoko Suzuki for their assistance for histopathological specimen preparation.

Conflict of interest---- The authors declare that there is no conflict of interest.

\section{REFERENCES}

Aroor, A.R., James, T.T., Jackson, D.E. and Shukla, S.D. (2010):
Differential changes in MAP kinases, histone modifications, and liver injury in rats acutely treated with ethanol. Alcohol. Clin. Exp. Res., 34, 1543-1551.

Badger, A.M., Bradbeer, J.N., Votta, B., Lee, J.C., Adams, J.L. and Griswold, D.E. (1996): Pharmacological profile of SB 203580, a selective inhibitor of cytokine suppressive binding protein/p38 kinase, in animal models of arthritis, bone resorption, endotoxin shock and immune function. J. Pharmacol. Exp. Ther., 279, 1453-1461.

Calder, P.C. (2009): Polyunsaturated fatty acids and inflammatory processes: New twists in an old tale. Biochimie, 91, 791-795.

Cao, W., Daniel, K.W., Robidoux, J., Puigserver, P., Medvedev, A.V., Bai, X., Floering, L.M., Spiegelman, B.M. and Collins, S. (2004): p38 mitogen-activated protein kinase is the central regulator of cyclic AMP-dependent transcription of the brown fat uncoupling protein 1 gene. Mol. Cell. Biol., 24, 3057-3067.

Chen, H.W., Lii, C.K., Ko, J.J., Wang, S.T. and Hsu, J.D. (1996): Regulatory effects of dietary n-3 and n-6 lipids on plasma and hepatic lipid levels, liver cell number and microsomal protein content in spontaneously hypertensive rats. Prostaglandins Leukot. Essent. Fatty Acids, 55, 329-335.

Chilakapati, J., Shankar, K., Korrapati, M.C., Hill, R.A. and Mehendale, H.M. (2005): Saturation toxicokinetics of thioacetamide: role in initiation of liver injury. Drug Metab. Dispos., 33, 1877-1885.

Cohen, J.C., Horton, J.D. and Hobbs, H.H. (2011): Human fatty liver disease: old questions and new insights. Science, 332, 15191523.

Cuenda, A. and Rousseau, S. (2007): p38 MAP-kinases pathway regulation, function and role in human diseases. Biochim. Biophys. Acta, 1773, 1358-1375.

Cunningham, F.M., Woollard, P.M. and Camp, R.D. (1985): Proinflammatory properties of unsaturated fatty acids and their monohydroxy metabolites. Prostaglandins, 30, 497-509.

Elabdeen, H.R., Mustafa, M., Szklenar, M., Rühl, R., Ali, R. and Bolstad, A.I. (2013): Ratio of pro-resolving and pro-inflammatory lipid mediator precursors as potential markers for aggressive periodontitis. PLoS One, 8, e70838.

Feranchak, A.P., Berl, T., Capasso, J., Wojtaszek, P.A., Han, J. and Fitz, J.G. (2001): p38 MAP kinase modulates liver cell volume through inhibition of membrane $\mathrm{Na}+$ permeability. J. Clin. Invest., 108, 1495-1504.

Iida, C., Fujii, K., Koga, E., Washino, Y., Kitamura, Y., Ichi, I., Abe, K., Matsura, T. and Kojo, S. (2009): Effect of alpha-tocopherol on carbon tetrachloride intoxication in the rat liver. Arch. Toxicol., 83, 477-483.

Imajo, K., Yoneda, M., Kessoku, T., Ogawa, Y., Maeda, S., Sumida, Y., Hyogo, H., Eguchi, Y., Wada, K. and Nakajima, A. (2013): Rodent models of nonalcoholic fatty liver disease/nonalcoholic steatohepatitis. Int. J. Mol. Sci., 14, 21833-21857.

Kalogeropoulos, N., Panagiotakos, D.B., Pitsavos, C., Chrysohoou, C., Rousinou, G., Toutouza, M. and Stefanadis, C. (2010): Unsaturated fatty acids are inversely associated and n-6/n-3 ratios are positively related to inflammation and coagulation markers in plasma of apparently healthy adults. Clin. Chim. Acta, 411, 584-591.

Kumar, S., Boehm, J. and Lee, J.C. (2003): p38 MAP kinases: key signalling molecules as therapeutic targets for inflammatory diseases. Nat. Rev. Drug Discov., 2, 717-726.

Lee, J.C., Kumar, S., Griswold, D.E., Underwood, D.C., Votta, B.J. and Adams, J.L. (2000): Inhibition of p38 MAP kinase as a therapeutic strategy. Immunopharmacology, 47, 185-201. 
Decreased p-p38 MAPK attenuated liver necrosis in obese mice

Lv, K.Y., Yu, X.Y., Bai, Y.S., Zhu, S.H., Tang, H.T., Ben, D.F., Xiao, S.C., Wang, G.Y., Ma, B. and Xia, Z.F. (2012): Role of inhibition of p38 mitogen-activated protein kinase in liver dysfunction after hemorrhagic shock and resuscitation. J. Surg. Res., 178, 827-832.

Pilkington, S.M., Rhodes, L.E., Al-Aasswad, N.M., Massey, K.A. and Nicolaou, A. (2014): Impact of EPA ingestion on COX- and LOX-mediated eicosanoid synthesis in skin with and without a pro-inflammatory UVR challenge--report of a randomised controlled study in humans. Mol. Nutr. Food Res., 58, 580-590.

Shirai, M., Arakawa, S., Miida, H., Matsuyama, T., Kinoshita, J., Makino, T., Kai, K. and Teranishi, M. (2013): Thioacetamide-induced hepatocellular necrosis is attenuated in diet-induced obese mice. J. Toxicol. Pathol., 26, 175-186.

Shirai, M., Matsuoka, M., Makino, T., Kai, K., Teranishi, M. and Takasaki, W. (2015): Hepatic glutathione contributes to attenuation of thioacetamide-induced hepatic necrosis due to suppression of oxidative stress in diet-induced obese mice. J. Toxicol. Sci., 40, 509-521.

Strassburg, K., Huijbrechts, A.M., Kortekaas, K.A., Lindeman, J.H., Pedersen, T.L., Dane, A., Berger, R., Brenkman, A., Hankemeier, T., van Duynhoven, J., Kalkhoven, E., Newman, J.W. and Vreeken, R.J. (2012): Quantitative profiling of oxylipins through comprehensive LC-MS/MS analysis: application in cardiac sur- gery. Anal. Bioanal. Chem., 404, 1413-1426.

Tam, V.C. (2013): Lipidomic profiling of bioactive lipids by mass spectrometry during microbial infections. Semin. Immunol., 25, 240-248.

Tibbles, L.A., Spurrell, J.C., Bowen, G.P., Liu, Q., Lam, M., Zaiss, A.K., Robbins, S.M., Hollenberg, M.D., Wickham, T.J. and Muruve, D.A. (2002): Activation of p38 and ERK signaling during adenovirus vector cell entry lead to expression of the C-X-C chemokine IP-10. J. Virol., 76, 1559-1568.

vom Dahl, S., Schliess, F., Graf, D. and Häussinger, D. (2001): Role of p38(MAPK) in cell volume regulation of perfused rat liver. Cell. Physiol. Biochem., 11, 285-294.

Wang, X., Lu, Y., Xie, B. and Cederbaum, A.I. (2009): Chronic ethanol feeding potentiates Fas Jo2-induced hepatotoxicity: role of CYP2E1 and TNF-alpha and activation of JNK and P38 MAP kinase. Free Radic. Biol. Med., 47, 518-528.

Wu, D. and Cederbaum, A. (2008): Cytochrome P4502E1 sensitizes to tumor necrosis factor alpha-induced liver injury through activation of mitogen-activated protein kinases in mice. Hepatology, 47, 1005-1017.

Zhang, L., Li, H.Z., Gong, X., Luo, F.L., Wang, B., Hu, N., Wang, C.D., Zhang, Z. and Wan, J.Y. (2010): Protective effects of Asiaticoside on acute liver injury induced by lipopolysaccharide/Dgalactosamine in mice. Phytomedicine, 17, 811-819. 